\title{
Use of a water-soluble form of cannabinol for the treatment of sleeplessness
}

\begin{abstract}
Introduction: Sleeplessness, i.e., getting <7hours sleep/night (L7H), causes significant health and financial problems for individuals and industry. Sleeplessness is classified into 4 categories: difficulty initiating sleep (DIS), difficulty maintaining sleep (DMS), early morning awakening (EMA), and nonrestorative sleep (NRS). This study surveyed individuals with sleeplessness who were taking a water-soluble product of cannabinol $(\mathrm{CBN})$ treated using nano technology, which has recently become available.
\end{abstract}

Methods: Individuals taking this product completed a self-evaluation survey prior to and after taking this product for at least 7days. The data was analyzed for changes in rate and severity of sleeplessness. Severity was assessed by the number of days/week for all categories except NRS, which had a symptom score with the range: -6 to +6 . Significance was $\mathrm{p}<0.001$

Results: Sixty individuals returned the survey. For all sleep disorder categories, using this product significantly decreased incidence (L7S: $42 \%$ to $13 \%$, DIS: $42 \%$ to $2 \%$, DMS: $57 \%$ to $22 \%$, EMA: $42 \%$ to $13 \%$, NRS: $60 \%$ to $13 \%$ ) and severity (L7S: $5.72 \pm 1.46$ to $1.88 \pm$ 2.80, DIS: $4.77 \pm 2.12$ to $0.38 \pm 1.27$, DMS: $5.00 \pm 1.89$ to $2.21 \pm 2.91$, EMA: $4.96 \pm 1.82$ to $1.7 \pm 2.76$, NRS: $-1.43+3.03$ to $2.85 \pm 2.16$ ). Thirteen individuals had an adverse side effect that resolved in all individuals after self-adjustment of the dose.

Conclusions: This nano treated $\mathrm{CBN}$ product rapidly induced sleep initially and after awakening, increasing the time and quality of sleep in most individuals suffering from sleeplessness.

Keywords: CBN, cannabinol, sleep, sleeplessness, nanotechnology
Volume I4 Issue 4 - 202I

\section{Robert Kaufmann \\ Department of Immunology, Allergy Midwest Allergy Sinus Asthma PC, USA}

Correspondence: Robert Kaufmann, Department of Immunology, Allergy Midwest Allergy Sinus Asthma PC, Director of Research, Shaman Botanicals LLC, USA, Tel 217-7|7-4404, Email robert@cbdamericanshaman.com

Received: June 05, 2021 | Published: July 12, 2021
Abbreviations: L7H, less than 7hours sleep/night; DIS, difficulty initiating sleep; DMS, difficulty maintaining sleep; EMA, early morning awakening; NRS, nonrestorative sleep; CBN, cannabinol

\section{Introduction}

Sleeplessness, defined as less than seven hours sleep at least two nights a week, affects almost everyone at some point. However, it is a chronic condition for up to $36 \%$ of the US population. It takes a staggering toll on the economy and personal health, accounting for $\$ 63.2$ billion of lost productivity annually in the US alone $\mathrm{e}^{1,2}$ and increasing the frequency, severity, and medical costs of personal injury. ${ }^{3,4}$ In addition, insufficient sleep duration is associated with serious medical conditions, including obesity, hypertension, hyperlipidemia, inflammation, heart attack, stroke, and diabetes; it results in a $12 \%$ increase in mortality. ${ }^{5-7}$

Sleeplessness can be classified into four distinct categories. ${ }^{8}$

1. Difficulty initiating sleep (DIS): Taking $>30$ minutes to fall asleep.

2. Difficulty maintaining sleep (DMS): Awakening and taking $>1$ hour before sleep resumed.

3. Early morning awakening (EMA): Awakening $>2$ hours before desired.

4. Nonrestorative sleep (NRS): Feeling unrefreshed upon awakening
While an individual may experience only one type of sleeplessness, many people experience combinations of more than one. ${ }^{8,9}$ This is especially true for NRS, as the regular transition of sleep stages (i.e., without excessive arousals or fragmentation) is needed for sleep to be "restorative." Most individuals must get at least seven hours of sleep each night to properly progress through these sleep transitions and must get it at least six days per week to maintain optimal health and functioning. ${ }^{5,10}$

In spite of the tremendous cost and all this knowledge, sleeplessness is consistently under-recognized, under-emphasized, and under-treated by the general population, health professionals, government agencies, employers, and insurance companies. As a result, few people seek medical assistance, health professionals often provide significant under-treatment, and many insurance plans do not cover either testing or treatment of this condition., ${ }^{2,11-13}$

Although the preferred method of treatment of sleep disorders is cognitive behavioral therapy, sleeplessness is most commonly treated with medications, either by medical professionals or via self-medication. ${ }^{2,4,12}$ The ideal substance to be taken to resolve occasional sleeplessness would have the following characteristics: can be administered orally, induces sleep rapidly, maintains sleep for between seven and nine hours, induces restorative sleep, is not habit forming, and has a low risk of overdose leading to death.

Cannabinol (CBN), in low doses, has relaxing and sedating effects in both humans and animals. ${ }^{16-21}$ The safety of CBN in humans is well established, it has no psychotropic activity at all when consumed 
orally by humans, and it has little to no adverse effects in doses up to 400mg. ${ }^{22-26}$

The vast majority of CBN products available in the US for sleep disorders are tinctures of $\mathrm{CBN}$. A water-soluble form of $\mathrm{CBN}$, created using nano technology, has recently become available to the general public as a nutritional supplement. This study consisted of a survey of the first individuals who started taking this product for occasional sleeplessness to test its effectiveness.

\section{Materials and methods}

The CBN nutritional supplement used in this study was derived exclusively from hemp plants and treated using proprietary nano technology to produce a water-soluble liquid. Individuals voluntarily bought and administered this product on their own and self-adjusted the amount of CBN nightly until they reached their desired sleep goals. Recommended initial amounts to be taken were $2 \mathrm{mg}$ for women and $3 \mathrm{mg}$ for men; however, individuals could talk as much or as little as they desired. Individuals obtaining the product for sleep disorders were asked if they would fill out a survey. The survey consisted of a series of questions that asked about sleep prior to starting the product and after taking the product. Respondents had to have been taking the product for a minimum of seven days before they could fill out the survey. The veracity of the responses was the responsibility of the respondents. This paper is the summary of the results of these respondents' surveys.

The survey questions asked if the respondent had been experiencing L7H, DIS, DMS, and/or EMA, and if so, the average number of nights per week this occurred. They were also asked if they experienced
NRS and, if so, to fill out a restorative sleep questionnaire to identify specific symptoms. The symptoms identified produced a score from -6 to 6 , with NRS symptoms being negative and restorative sleep symptoms being positive. Because NRS symptoms can exist whether an individual has a specific sleep disorder or not, questions were asked about age, sex, amount being taken, side effects, and whether the respondent had needed to vary the amount being taken.

Statistical analysis on continuous variables was performed using paired t-tests and on non-continuous paired variables using a Chisquare test. The number of days that L7H, DIS, DMS, and EMA had occurred was analyzed only for those affected by that disorder. In addition, the total number of days per week that all sleep disorders occurred prior to and after using $\mathrm{CBN}$ was calculated for each affected individual. NRS scores were calculated initially on only those individuals suffering from NRS prior to CBN. However, since NRS can occur with or without a specific sleep disorder, NRS score analysis was also performed using all survey respondents.

\section{Results}

Of the 60 individuals who responded to the survey, 24 were female and 36 male. The average age was $42.9 \pm 12.8$ years, with no significant difference between the females $(45.0 \pm 12.5)$ and males $(41.5 \pm 13.0)$.

Table 1 shows that $\mathrm{CBN}$ significantly decreased the number of individuals experiencing each type of sleep difficulty. It dropped $\mathrm{L} 7 \mathrm{H}$ from 25 individuals to 8 individuals and decreased DIS from $43 \%$ to $2 \%$, DMS from $57 \%$ to $22 \%$, EMA from $43 \%$ to $15 \%$, and NRS from $60 \%$ to $13 \%$. The $p$ values for the paired $t$ tests were all $<0.001$.

Table I Number and prevalence of various sleep disorders before and after CBN treatment

\begin{tabular}{lllll}
\hline Sleep Disorder & Survey Answer & Prior to CBN & After CBN & p-value \\
\hline \multirow{2}{*}{ L7H } & Yes & $25(42 \%)$ & $8(13 \%)$ & \\
& No & $35(58 \%)$ & $52(87 \%)$ & $<0.00$ I \\
\multirow{2}{*}{ DIS } & Yes & $26(43 \%)$ & $1(2 \%)$ & \\
& No & $34(57 \%)$ & $59(98 \%)$ & $<0.00$ I \\
\multirow{2}{*}{ DMS } & Yes & $34(57 \%)$ & $13(22 \%)$ & \\
& No & $26(43 \%)$ & $47(78 \%)$ & $<0.00$ I \\
EMA & Yes & $26(43 \%)$ & $8(13 \%)$ & \\
& No & $34(57 \%)$ & $52(87 \%)$ & $<0.001$ \\
\multirow{2}{*}{ NRS } & Yes & $36(60 \%)$ & $8(13 \%)$ & \\
& No & $24(40 \%)$ & $52(87 \%)$ & $<0.00$ I \\
\hline
\end{tabular}

The CBN product used in this study significantly decreased the percentage of individuals suffering from every type of sleep disorder. A "Yes" answer signifies that the individual was suffering from that sleep disorder and a "No" means they were not.

Abbreviations: Less than 7hours sleep/night (L7H), Difficulty initiating sleep (DIS), Difficulty maintaining sleep (DMS), Early morning awakening (EMA), Nonrestorative sleep (NRS), Cannabinol (CBN)

None of the 26 individuals with DIS prior to starting CBN were still experiencing DIS after starting CBN. Three (3) individuals with DIS after starting CBN reported that $\mathrm{CBN}$ had caused DIS from the adverse side effect of restlessness. However, two of them decreased the amount being taken from $4 \mathrm{mg}$ to $1 \mathrm{mg}$ and the restlessness did not occur. One of the individuals who had DMS prior to starting CBN continued to have DMS occasionally after taking $2 \mathrm{mg}$ of CBN before bedtime. This individual found that taking an additional $1 \mathrm{mg}$ when she awoke during the night facilitated an immediate return to sleep.
However, taking $3 \mathrm{mg}$ before bedtime resulted in a feeling of being "too wired," causing DIS.

Table 2 compares the number of days per week that individuals reported the various sleep disorders prior to and after CBN. As can be seen, the mean number of days of each sleep disorder decreased significantly with $\mathrm{CBN}$ administration. For those reporting $\mathrm{L} 7 \mathrm{H}$, the average number of days per week decreased by 3.84 days per week from 5.72 to 1.88 . The greatest change in sleeplessness was reported 
by those suffering from DIS: the average days per week decreased almost entirely, from 4.77 to 0.38 .

The average NRS score for all survey respondents increased from $-1.43 \pm 3.03$ to $2.85 \pm 2.16(p<0.001)$. This improvement in NRS

Table 2 Number of days per week individuals experienced a sleep disorder scores was due almost exclusively to those suffering from NRS prior to starting CBN $(\mathrm{n}=36)$, as their scores improved from $-2.42 \pm 1.93$ to $2.75 \pm 3.01(p<0.001)$. The total number of days per week that all sleep disorders occurred decreased significantly from $9.27 \pm 9.57$ to $2.98 \pm 6.11(p<0.001)$.

\begin{tabular}{|c|c|c|c|c|c|c|}
\hline \multirow{2}{*}{ Sleep Disorder } & \multirow{2}{*}{ Number of Individuals } & \multicolumn{2}{|l|}{ Prior to $\mathrm{CBN}$} & \multicolumn{2}{|l|}{ After CBN } & \multirow{2}{*}{$p$ value } \\
\hline & & Mean (days) & s.d. & Mean (days) & s.d. & \\
\hline L7H & 25 & 5.72 & 1.46 & 1.88 & 2.8 & $<0.001$ \\
\hline DIS & 26 & 4.77 & 2.12 & 0.38 & 1.27 & $<0.001$ \\
\hline DMS & 34 & 5.00 & 1.89 & 2.21 & 2.91 & $<0.001$ \\
\hline EMA & 26 & 4.96 & 1.82 & 1.77 & 2.76 & $<0.001$ \\
\hline
\end{tabular}

The CBN product used in this study significantly decreased mean number of days per week that individuals suffered from every type of sleep disorder. Abbreviations: Less than 7hours sleep/night (L7H), Difficulty initiating sleep (DIS), Difficulty maintaining sleep (DMS), Early morning awakening (EMA), Nonrestorative sleep (NRS), Cannabinol (CBN), Standard deviation (s.d.)

The CBN dosages regularly used by individuals are displayed in Figure 1; two individuals (one male and one female) did not report how much $\mathrm{CBN}$ they had been taking. The majority (77.5\%) of survey respondents reported taking $\geq 0.5 \mathrm{ml}$, which is equivalent to $\geq 2 \mathrm{mg}$. On average, males took more CBN (mean of $2.94 \pm 1.04, \mathrm{n}=35$ ) than did females (mean of $2.02 \pm 1.12, \mathrm{n}=23$ ), a significant difference $(\mathrm{p}<0.005)$. Overall, $48.3 \%$ of the individuals had to adjust their CBN nightly dosage to achieve optimal sleep. The percentage of women who adjusted their amount (50\%) was essentially the same as that of the men $(47.2 \%)$. Nine individuals found that they did not have to use CBN every night; however, if they went several nights without it, their sleep issues would return.

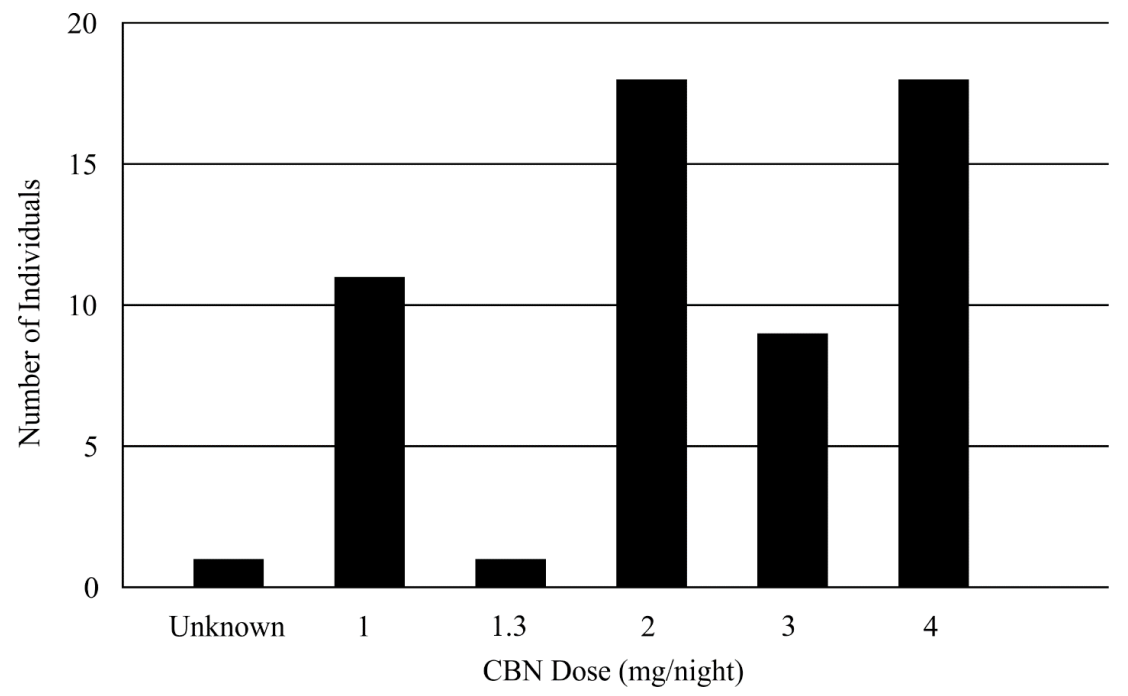

Figure I Number of Individuals taking Different Doses of CBN.

Fourteen individuals had been taking at least one of 21 other medications for sleep: nine were taking $\mathrm{CBD}$, five were taking diphenhydramine, five were taking melatonin, three were taking chamomile, and five were taking various other prescription medications. After starting CBN, ten individuals $(71.4 \%)$ were able to discontinue a total of 21 of these medications, with $8(57 \%)$ being able to eliminate all of their other sleep medications; the other two were able to reduce from four and two medications to one medication each (chamomile and Xanax, respectively). The remaining four individuals were each on one medication (two melatonin and two CBD) and continued on that medication.

Thirteen individuals (six women and seven men) reported adverse side effects, the most common being the feeling of grogginess in the morning (five individuals). However, the morning grogginess resolved when they reduced the amount being taken. Three individuals reported vivid dreams, but these similarly resolved with decreasing the amount being taken. Three individuals reported that they experienced restlessness and/or mind racing after taking CBN. Two of these individuals decreased the amount of CBD from $4 \mathrm{mg}$ to $1 \mathrm{mg}$, and the restlessness did not occur. The remaining individual was taking 4 $\mathrm{mg} / \mathrm{night}$ and did not try decreasing the dose but just stopped taking CBN. Two individuals reported morning headaches but a resolution of their sleep disorders; when the amount being taken was decreased, the headaches resolved and the sleeplessness did not return. Overall, in 12 of the 13 individuals who reported adverse side effects, the side effects were apparently due to taking excess amounts of CBN. In the remaining individual, the side effect may have resolved if they had decreased the amount being taken from $4 \mathrm{mg} / \mathrm{night}$ to something less, however, they did not try this, so the cause is unknown. Therefore, 
the rate that adverse side effects necessitated discontinuing the use of CBN was $1.7 \%(1 / 60)$. In contrast, the remaining 59 of the 60 individuals reported that their sleep had improved dramatically and that they were continuing to take $\mathrm{CBN}$ to resolve their sleep issues, yielding an overall satisfaction rate of $98.3 \%$.

Other beneficial effects were also reported. Fifteen reported having good dreaming experiences that were absent prior. Two reported maintenance of pain relief. One individual each reported an increased libido and no anxiety the next day. An individual with cerebral palsy reported muscle relaxation the next day.

\section{Discussion}

This study found that very low doses $(1-4 \mathrm{mg})$ of a nano treated, water-soluble CBN product were successful in significantly decreasing the incidence and severity of sleeplessness overall and in each of the four distinct categories of sleeplessness. The greatest benefit of this product was the ability to induce sleep and to either keep individuals asleep or to return them to sleep after awakening during the night. In addition, this CBN product appears to have allowed individuals to progress normally through the various stages of sleep, including REM sleep as evidenced by the reduction of NRS incidence and that many individuals reporting significantly enhanced dreaming.

Almost half of the individuals had to make adjustments in the amount of CBN being taken. Once the proper amount of CBD was found for each individual, this product induced sleep for $98 \%$ and maintained sleep for the majority but not all of the participants. It is well established that the absorption and metabolism of cannabinoids can vary greatly, ${ }^{27,28}$ thus, some individuals may need a "booster" during the night if they have DMS.

Interestingly, apparently excess amounts of this product resulted in restlessness in some individuals and in next-day drowsiness others, while these same levels did not result in any adverse effects for most individuals. Both the restlessness and drowsiness were dose related and resolved with self-adjustment of the dose. The side effect of dose related restlessness and extreme drowsiness is a new finding for studies of CBN. No mention of restlessness with CBN has never been reported. Low dose $\mathrm{CBN}$ has been reported to have only a mild sedative effect in humans ${ }^{20,21}$ and the sedative effect of CBN decreased with increasing dosage. ${ }^{16}$ No studies have reported excessive drowsiness or other sedative effect as long lasting as was found with this product.

The mechanism by which CBN causes drowsiness is unknown, although it does not appear to be related to how melatonin induces sleep. ${ }^{29}$ Rather, it may be related to the fact that, like most phytocannabinoids, $\mathrm{CBN}$ binds to multiple receptors (i.e., $\mathrm{CB} 1, \mathrm{CB} 2$, TRPV, etc. ${ }^{19,30}$ ) and the products of CBN metabolism may be binding and affect the receptor response also. This differential in binding is a plausible reason why cannabinoids can exert differing effects at differing times and levels of dosing. Given the small amounts of CBN (1-4mg) taken in this study, the specific product's bioavailability would appear to be substantially greater than that of tinctures of CBN, since only minor drowsiness has been reported to occur with $\mathrm{CBN}$ tinctures at doses of $10-20 \mathrm{mg} .{ }^{21}$

It is possible that the nano treatment of $\mathrm{CBN}$ in this case is changing not only the bioavailability but also the mechanism of absorption. Nano treatment of cannabidiol (CBD) has been shown to significantly increase the bioavailability which may be due to the majority of the CBD being absorbed via the intestinal lymphatic system rather than the portal system. ${ }^{31,32}$ Lymphatic absorption of CBD would avoid the "first-pass" metabolism possibly resulting in a difference in ratio of the blood levels of CBD to its metabolites, which could result possibly explain the previously unreported effects of $\mathrm{CBN}$ found in this study.

\section{Conclusion}

This study found that low doses (1-4mg) of a nano treated, watersoluble CBN product significantly improved the sleep of individuals who had been experiencing occasional sleeplessness. It rapidly induced sleep initially and after awakening during the night. It improved both the length and quality of sleep such that the individuals felt rested upon awakening in the morning. It appeared to produce restful sleep by enabling individuals to progress through the various stages of sleep normally.

Notably, almost half (48\%) of the individuals reported needing to adjust the CBN dosage to achieve optimal sleep, whether from not taking enough CBN (17) or taking too much (12). All reported adverse side effects seemed to have been caused by taking too much $\mathrm{CBN}$, as they resolved completely when the dosage was decreased or discontinued. This is the first time prolonged drowsiness after awakening has been reported with increasing amounts of $\mathrm{CBN}$, which may be due to differences in intestinal absorption secondary to the nano processing of the $\mathrm{CBN}$.

\section{Acknowledgments}

None.

\section{Conflicts of interest}

Dr. Kaufmann is Director of Research for Shaman Botanicals, LLC declares no conflicts of interest.

\section{Funding}

No funding sources.

\section{References}

1. Kessler RC, Berglund, PA, Coulouvrat, C, et al. Insomnia and the performance of US workers: results from the America insomnia survey. Sleep. 2011;34(9):1161-1171.

2. Kraus SS, Rabin LA. Sleep America: managing the crisis of adult chronic insomnia and associated conditions. J Affect Disord. 2012;138(3):192212.

3. Kessler RC, Berglund PA, Coulouvrat C, et al. Insomnia, comorbidity, and risk of injury among insured Americans: results from the America Insomnia Survey. Sleep. 2012;35(6):825-834.

4. Shahly V, Berglund PA, Coulouvrat C, et al. The associations of insomnia with costly workplace accidents and errors: results from the America Insomnia Survey. Arch Gen Psychiatry. 2012;69(10):1054-1063.

5. Grandner MA, Alfonso-Miller P, Fernandez-Mendoza J, et al. Sleep: important considerations for the prevention of cardiovascular disease. Curr Opin Cardiol. 2016;31(5):551-565.

6. Cappuccio F, Miller M. Sleep and cardio-metabolic disease. Curr Cardiol Rep. 2017;19:110

7. Cappuccio FP, D’Elia L, Strazzullo P, et al. Sleep duration and all-cause mortality: a systematic review and meta-analysis of prospective studies. Sleep. 2010;33:585-592.

8. Roth T, Zammit G, Lankford A, et al. Nonrestorative sleep as a distinct component of insomnia. Sleep. 2010;33(4):449-458.

9. Hohagen F, Käppler C, Schramm E, et al. Sleep onset insomnia, sleep maintaining insomnia and insomnia with early morning awakeningtemporal stability of subtypes in a longitudinal study on general practice attenders. Sleep. 1994;17(6):551-554. 
10. Sarsour K, Van Brunt DL, Johnston JA, etal. Associations of nonrestorative sleep with insomnia, depression, and daytime function. Sleep Med. 2010;11(10):965-972.

11. Jackson CL, Redline S, Emmons KM. Sleep as a potential fundamental contributor to disparities in cardiovascular health. Annu Rev Public Health. 2015;36:417-440.

12. Morin CM, LeBlanc M, Bélanger L, et al. Prevalence of insomnia and its treatment in Canada. Can J Psychiatry. 2011;56(9):540-548.

13. Parish JM, Freedman NS, Manaker S. Evolution in reimbursement for sleep studies and sleep centers. Chest. 2015;147(3):600-606.

14. van Straten A, van der Zweerde T, Kleiboer A, et al. Cognitive and behavioral therapies in the treatment of insomnia: A meta-analysis. Sleep Med Rev. 2018;38:3-16.

15. Yoshida H, Usami N, Ohishi Y, et al. Synthesis and pharmacological effects in mice of halogenated cannabinol derivatives. Chem Pharm Bull (Tokyo). 1995;43(2):335-337.

16. Loewe S. Studies on the pharmacology of marihuana. In: The Mayor's Committee on Marihuana. The Marihuana Problems in the City of New York. Lancaster (PA): The Jaques Cattell Press; 1994. p. 149-212.

17. Takahashi RN, Karniol IG. Pharmacologic interaction between cannabinol and delta9-tetrahydrocannabinol. Psychopharmacologia. 1975;41(3):277-284.

18. Oishi R, Itoh Y, Nishibori $\mathrm{M}$, et al. Enhancement by alphafluoromethylhistidine of the thiopental sleep-prolonging action of delta 9-tetrahydrocannabinol. Psychopharmacology (Berl). 1998;95(1):77-81.

19. Rhee MH, Vogel Z, Barg J, et al. Cannabinol derivatives: binding to cannabinoid receptors and inhibition of adenylylcyclase. J Med Chem. 1997;40(20):3228-3233.

20. Binet L, Binet P, Miocque M, et al. Pharmacodynamic properties (sedative and spasmolytic action) of some aliphatic terpenic alcohols. Ann Pharm Fr. 1972;30: 611-616.

21. Musty RE, Karniol IG, Shirikawa I, et al. Interactions of delta-9tetrahydrocannabinol and cannabinol in man. In: Braude MC, Szara S, editors. The Pharmacology of Marihuana. New York (NY): Raven Press; 1976. p. 559-563.

22. Hollister LE. Cannabidiol and cannabinol in man. Experientia. 1973;29(7):825-826
23. Hollister LE, Gillespie $H$. Interations in man of delta-9tetrahydrocannabinol. II. Cannabinol and cannabidiol. Clin Pharmacol Ther. 1975;18(1):80-83.

24. Karniol IG, Shirakawa I, Takahashi RN, et al. Effects of delta9tetrahydrocannabinol and cannabinol in man. Pharmacology. 1975;13(6):502-512.

25. Perez-Reyes $\mathrm{M}$, Timmons $\mathrm{MC}$, Davis $\mathrm{KH}$, et al. A comparison of the pharmacological activity in man of intravenously administered delta9-tetrahydrocannabinol, cannabinol, and cannabidiol. Experientia. 1973;29(11):1368-1369.

26. Cohen K, Weinstein A. Synthetic and Non-synthetic cannabinoid drugs and their adverse effects-A review from public health prospective. Front Public Health. 2018;6:162.

27. Guy GW, Flint ME. A Single Centre, Placebo-Controlled, Four Period, Crossover, Tolerability Study Assessing, Pharmacodynamic Effects, Pharmacokinetic Characteristics and Cognitive Profiles of a Single Dose of Three Formulations of Cannabis Based Medicine Extracts (CBMEs) (GWPD9901), Plus a Two Period Tolerability Study Comparing Pharmacodynamic Effects and Pharmacokinetic Characteristics of a Single Dose of a Cannabis Based Medicine Extract Given via Two Administration Routes (GWPD9901 EXT). J Cannabis Therapeutics. 2004;3(3):35-77.

28. Huestis M. Human cannabinoid pharmacokinetics. Chem Biodivers. 2007;4(8):1770-1804.

29. Sugden D. Sedative potency and 2-[125I]iodomelatonin binding affinity of melatonin analogues. Psychopharmacology (Berl). 1995;117(3):364370 .

30. Qin N, Neeper MP, Liu Y, et al. TRPV2 is activated by cannabidiol and mediates CGRP release in cultured rat dorsal root ganglion neurons. $J$ Neurosci. 2008;28(24):6231-6238.

31. Atsmon J, Cherniakov I, Izgelov D, et al. PTL401, a New Formulation Based on Pro-Nano Dispersion Technology, Improves Oral Cannabinoids Bioavailability in Healthy Volunteers. J Pharm Sci. 2018;107(5):14231429.

32. Zgair A, Lee JB, Wong JCM, et al. Oral administration of cannabis with lipids leads to high levels of cannabinoids in the intestinal lymphatic system and prominent immunomodulation. Sci Rep.2017;7(1):14542. 